\title{
CHARACTERISATION OF A PAPAIN-LIKE PROTEINASE DOMAIN ENCODED BY ORF1a OF THE CORONAVIRUS IBV AND DETERMINATION OF THE C-TERMINAL CLEAVAGE SITE OF AN 87 kDa PROTEIN
}

\author{
K. P. Lim and D. X. Liu \\ Institute of Molecular Agrobiology \\ 59A The Fleming \\ 1 Science Park Drive \\ Singapore 118240
}

\section{ABSTRACT}

Our previous studies have shown that two overlapping papain-like proteinase domains (PLPDs) encoded by the IBV sequence from nucleotides 4155 to 5550 is responsible for cleavage of the ORF 1a polyprotein to an $87 \mathrm{kDa}$ protein. In this study, we demonstrate that only the more 5' one of the two domains, PLPD-1 encoded between nucleotides 4155 and 5031 , is required for processing to the $87 \mathrm{kDa}$ protein. Site-directed mutagenesis studies have shown that the $\mathrm{Cys}^{1274}$ and $\mathrm{His}^{1435}$ residues are essential for the PLPD-1 activity, suggesting that they may be the components of the catalytic centre of this proteinase. Coexpression and immunoprecipitation studies have further revealed that PLPD can interact with the $87 \mathrm{kDa}$ protein. Meanwhile, data obtained from the construction and expression of a series of deletion mutants have indicated that the $87 \mathrm{kDa}$ protein is encoded by the $5^{\prime}-$ most 2600 bp part of ORF1a. Further deletion and mutagenesis studies are underway to determine precisely the C-terminal cleavage site of the $87 \mathrm{kDa}$ protein.

\section{INTRODUCTION}

Proteolytic processing of the $741 \mathrm{kDa}$ polyprotein encoded by mRNA 1 is one of the strategies employed by the Coronavirus IBV in replication of its genome. Two overlapping viral papain-like proteinase domains (PLPDs) belonging to the class of cysteine pro- 
teinases and a trypsin-like proteinase domain belonging to the picornavirus $3 \mathrm{C}$ proteinase supergroup are predicted to be encoded by the IBV sequence from nucleotide 4155 to 5550 and from 8864 to 9787 , respectively (Gorbalenya et al., 1989). The two overlapping PLPDs have been demonstrated to be responsible for cleavage of the 1a polyprotein to a novel $87 \mathrm{kDa}$ protein, as the release of this $87 \mathrm{kDa}$ product was observed only when the IBV sequence from nucleotide 4859 to 5753 was included in the expression plasmids (Liu et al., 1995).

In this communication we report that only the first PLPD encoded between nucleotides 4242 and 5031 is required for cis-cleavage of the $87 \mathrm{kDa}$ protein at its C-terminus. Substitution of either of the two putative core residues, Cys ${ }^{1274}$ and $\mathrm{His}^{1435}$, totally abolished the proteinase activity, thereby allowing us to conclude that they may be components of the catalytic centre of PLPD-1. The C-terminal cleavage site of the $87 \mathrm{kDa}$ protein was meanwhile determined by construction and expression of a series of deletion constructs. It was shown that the C-terminal scissile bond of the $87 \mathrm{kDa}$ protein was most likely located in the vicinity of $\mathrm{Gly}^{673}$ residue. In addition, evidence presented indicated that the $87 \mathrm{kDa}$ protein may interact with products encoded by the IBV sequence from nucleotide 2548 to 5753 .

\section{METHODS AND MATERIALS}

\subsection{In Vivo Expression of IBV Sequences}

IBV sequences placed under the control of a $\mathrm{T} 7$ promoter were transiently expressed in Vero cells using the vaccinia recombinant virus-T7 expression system as described before (Liu et al., 1994).

\subsection{Polymerase Chain Reaction}

Amplification reactions of the respective template DNAs with appropriate primers were performed with Native Pfu DNA polymerase (Stratagene) under standard buffer conditions with $2 \mathrm{mM} \mathrm{MgCl}$.

\subsection{Site-Directed Mutagenesis Studies}

Site-directed mutagenesis was performed as previously described (Liu et al., 1997).

\subsection{Radioimmunoprecipitation}

Plasmid DNA-transfected Vero cells were lysed with RIPA buffer $(50 \mathrm{mM}$ Tris $\mathrm{HCl}$, $\mathrm{pH} 7.5,150 \mathrm{mM} \mathrm{NaCl}, 1 \%$ sodium deoxycholate, $0.1 \%$ SDS) and precleared by centrifugation at $12,000 \mathrm{rpm}$ for 5 minutes at $4^{\circ} \mathrm{C}$. Immunoprecipitation was performed as described previously (Liu et al., 1994).

\subsection{Cell-Free Transcription and Translation}

Plasmid DNA was transcribed and translated in vitro with the TnT T7-coupled reticulocyte lysate system (TnT system) according to the instructions of the manufacturer (Promega). Reaction products were separated by SDS-PAGE and detected by autoradiography. 


\subsection{SDS-Polyacrylamide Gel Electrophoresis}

SDS-polyacrylamide gel electrophoresis (SDS-PAGE) of viral polypeptides was performed using $10 \%$ polyacrylamide gels (Laemmli, 1970). The $\left[{ }^{35} \mathrm{~S}\right]$-labelled polypeptides were detected by autoradiography of the dried gels.

\subsection{Construction of Plasmids}

Plasmid pIBV1a2 (formerly referred to as pKT1a2) which covers the IBV sequence from nucleotide 362 to 5753 (Liu et al., 1995), was used to construct pIBVla2 $\Delta 1$, pIBV1a2 $\Delta 4$, pIBV1a2 $\Delta 5$ and pIBV1a2 $\Delta 8$. Plasmid pIBV1a $2 \Delta 1$ which covers the IBV sequence from nucleotide 1311 to 5753 , was constructed by cloning a BamHI/MluI digested-PCR fragment into BglII/MluI digested pIBV1a2. BglII digests pIBV1a2 at a position immediately upstream of the viral sequence and MluI digests the IBV sequence at nucleotide position 3997. An artificial AUG initiation codon in an optimal context (AC$\mathrm{C} A U G \mathrm{G}$ ) was introduced immediately upstream of the viral sequence. The sequences of the upstream primer (LDX-3) and downstream primer (LDX-4) used for the construction of this plasmid are indicated in Table 1 . Plasmid pIBVla2 $\Delta 4$ was constructed by cloning a $2.7 \mathrm{~kb}$ BamHI/MluI digested PCR fragment containing the IBV sequence from nucleotides 362 to 3047 into BglII/MluI digested pIBV1a2, resulting in the deletion of the region between nucleotides 3047 and 3997. The upstream and downstream primers used are LKP-1 and LKP-2 respectively (Table 1 ). Plasmid pIBV1a $2 \Delta 5$ contains the IBV sequence from nucleotide 362 to 5027 . It was constructed by ligation of a $1.03 \mathrm{~kb}$ DNA fragment, obtained by digestion of pIBV1a2 with MluI and SpeI, into MluI/XbaI digested pIBVla1 (Liu et al., 1995). SpeI cuts the IBV sequence at nucleotide position 5027.A second series of deletion mutants was made by introducing a UAA termination codon into different positions of the viral sequences. The first plasmid, pIBV $1 \mathrm{a} 2 \Delta 8$ which contains a stop codon at the nucleotide position 2856, was constructed by cloning a BamHI/MluI digested PCR fragment into BglII/MluI digested pIBV1a2. The upstream primer was LKP-1 and the downstream primer was LKP1a2 $\Delta 8$. Plasmids pIBV1a2 $\Delta 10$, pIBV1a2 $\Delta 11$, and pIBV1a2 $\Delta 12$ contain an UAA codon at nucleotide positions 2601,2547 and 2517 respectively. The primers used to introduce these mutations are LKP1a2 $\Delta 10$, LKP1a2 $\Delta 11$ and

Table 1. Primers used for PCR amplification and site-directed mutagenesis

\begin{tabular}{|c|c|c|c|}
\hline Primer name & & Nucleotide sequences & ORF la position \\
\hline \multicolumn{4}{|c|}{ Deletion mutagensis primers } \\
\hline LDX-3 & $5^{\prime}$ & - ACG (CGGATCCAC) CATGGGTTCTAAG - $3^{\prime}$ & $1304-1323$ \\
\hline LDX-4 & $5^{\prime}$ & - TCTGTTTGCAAGTTACATCG - $3^{\prime}$ & $4060-4041$ \\
\hline LKP-1 & $5^{\prime}$ & - (TCTCAGGGA) TCCCCCCACATACC - $3^{\prime}$ & $370-383$ \\
\hline LKP-2 & $5^{\prime}$ & - ACGCTA (ACGCGT) TCATCAAGAGGCAG - $3^{\prime}$ & $3047-3022$ \\
\hline LKP $1 \mathrm{a} 2 \Delta 8$ & $5^{\prime}$ & - CTCTCATAGACGCGTTAGATCAAATGGC - $3^{\prime}$ & $2870-2843$ \\
\hline LKP $1 \mathrm{a} 2 \Delta 10$ & $5^{\prime}$ & -ATAGG (CCCGGGTTA) AGGTGGTATCT - $3^{\prime}$ & $2612-2584$ \\
\hline LKP1a2 $\Delta 11$ & $5^{\prime}$ & - CAGT (CCCGGGTTA) TGCTTTGCAAACCAC - 3' & $2557-2530$ \\
\hline LKP1a2 $\Delta 12$ & $5^{\prime}$ & - CATTATT (CCCGGGTTA) TTGAGACATTGGTG - 3' & $2530-2501$ \\
\hline \multicolumn{4}{|c|}{$\begin{array}{l}\text { Catalytic residue mutagenesis } \\
\text { primers }\end{array}$} \\
\hline $\mathrm{LKP} 1 \mathrm{a} 2 \Delta 4^{\mathrm{C} 1274 \cdot \mathrm{S}}$ & $5^{\prime}$ & - CGATGGAAAT (AGC) TGGATTAGTTCAGC- $3^{\prime}$ & $4338-4364$ \\
\hline $\mathrm{LKP} 1 \mathrm{a} 2 \Delta 4^{\mathrm{H} 1435-\mathrm{K}}$ & $5^{\prime}$ & - CTAATAGTGGC (AAG) TGTTATACACAAGC - $3^{\prime}$ & $4826-4853$ \\
\hline
\end{tabular}


LKP1a2 12 . The PCR fragments obtained for these constructs were digested with BamHI and SmaI, and then cloned into BglII/SmaI-digested pKT0 (Liu et al., 1994). Two mutants with alterations at the putative catalytic residues Cys ${ }^{1274}\left(\mathrm{pIBVla}_{2} \Delta 4^{\mathrm{C} 1274-\mathrm{S}}\right.$ ) and $\mathrm{His}^{1435}$ $\left(\mathrm{pIBV} 1 \mathrm{a} 2 \Delta 4^{\mathrm{H} 1435-\mathrm{K}}\right.$ ) were created by PCR amplification using pIBV la $2 \Delta 4$ as templates. The respective mutation primers used were LKP1a2 $\Delta 4^{\mathrm{C1274-S}}$ and LKP1a2 $\Delta^{\mathrm{H} 1435-\mathrm{K}}$ (Table 1). All constructs made by site-directed mutagenesis were selected and confirmed by automated sequencing.

Regions of the IBV sequences presented in these constructs are illustrated in Fig. 1.

\section{RESULTS}

\subsection{Further Analysis of the Involvement of the Two Papain-Like Proteinase Domains in Processing of the 1a Polyprotein to the 87 kDa Protein}

It was previously shown that when a construct (pIBV1a1) containing the IBV sequence from nucleotide 362 to 4858 was expressed, only the full-length product of $220 \mathrm{kDa}$ was observed (Liu et al., 1995). However, expression of the IBV sequence up to nucleotide 5753 (pIBV1a2), resulted in the detection of three protein species: a $250 \mathrm{kDa}$ full-length product and two cleavage products of $160 \mathrm{kDa}$ and $87 \mathrm{kDa}$ (Liu et al., 1995), implying that processing of the full length polypeptide had occurred. These results suggest strongly that the region

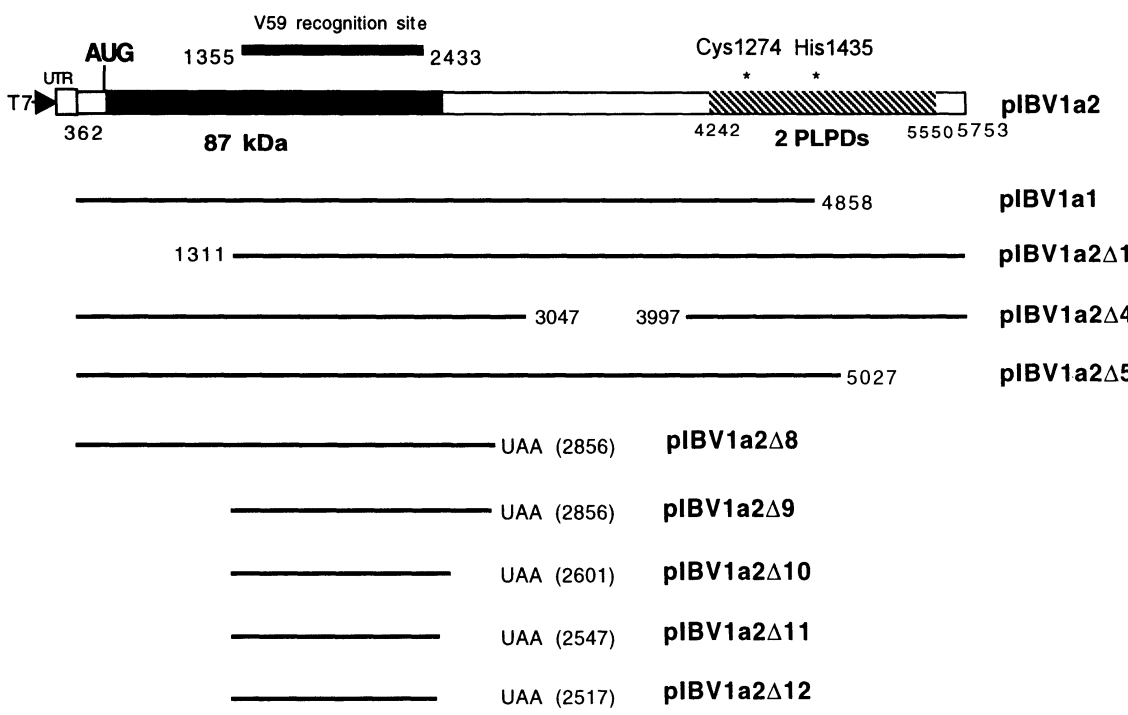

Figure 1. Diagram of the ORF1a region presented in plasmid pIBV1a2, showing the locations of the $87 \mathrm{kDa}$ protein, the two PLPDs and the region recognised by antiserum V59. The positions of the putative C-terminal cleavage site (Gly ${ }^{673} \downarrow$ Gly $^{674}$ dipeptide) of the $87 \mathrm{kDa}$ protein and the catalytic residues of the PLPD-1 (Cys ${ }^{1274}$ and $\mathrm{His}^{1435}$ ) are also indicated. Also shown are the regions of ORF1a present in plasmids pIBVlal and the various deletion mutants. The nucleotide positions of the UAA termination codon introduced are indicated in the brackets. 
from nucleotide 4859 to 5753 is essential for the processing of the 1a polyprotein to the 87 $\mathrm{kDa}$ protein. As computer prediction indicates that two putative overlapping PLPDs may be encoded from nucleotide 4155 to 5550 (Lee et al., 1991), it is highly likely that this cleavage is mediated by the two proteinase domains. However, it was not clear if both domains were required for the cleavage. To clarify this issue, plasmid pIBVla2 $\Delta 5$ (Fig. 1) which contains the IBV sequence from nucleotide 362 to 5027 was constructed and expressed in Vero cells using the vaccinia virus-T7 expression system (Fuerst, 1986). The deletion showed little, if any, effect on the processing to the $87 \mathrm{kDa}$ protein. As can be seen from Figure 2, expression of pIBV1a2 $\Delta 5$ resulted in the detection of the $87 \mathrm{kDa}$ protein as well as the full length 225 $\mathrm{kDa}$ protein and $136 \mathrm{kDa}$ cleavage product. This data indicates that only the first PLPD is responsible for the release of the $87 \mathrm{kDa}$ protein from its precursor.

\subsection{Mutational Analysis of the Putative Catalytic Dyad of PLPD-1}

Data presented above demonstrated that only the first of the two overlapping papainlike proteinase domains is required for processing of the la polyprotein to the $87 \mathrm{kDa}$

\footnotetext{
Figure 2. In vivo expression of pIBVla2 and pIBV1a2 $\Delta 5$ in Vero cells using the vaccinia recombinant virus-T7 expression system. The transfected cells were labelled with $\left[{ }^{35} \mathrm{~S}\right]$ methionine and the cell lysates were subjected to immunoprecipitation with antiserum V59 as indicated above each lane. SDSPAGE was performed on an SDS- $10 \%$ polyacrylamide gel. HMW-high molecular weight markers (numbers indicate kilodaltons).
}

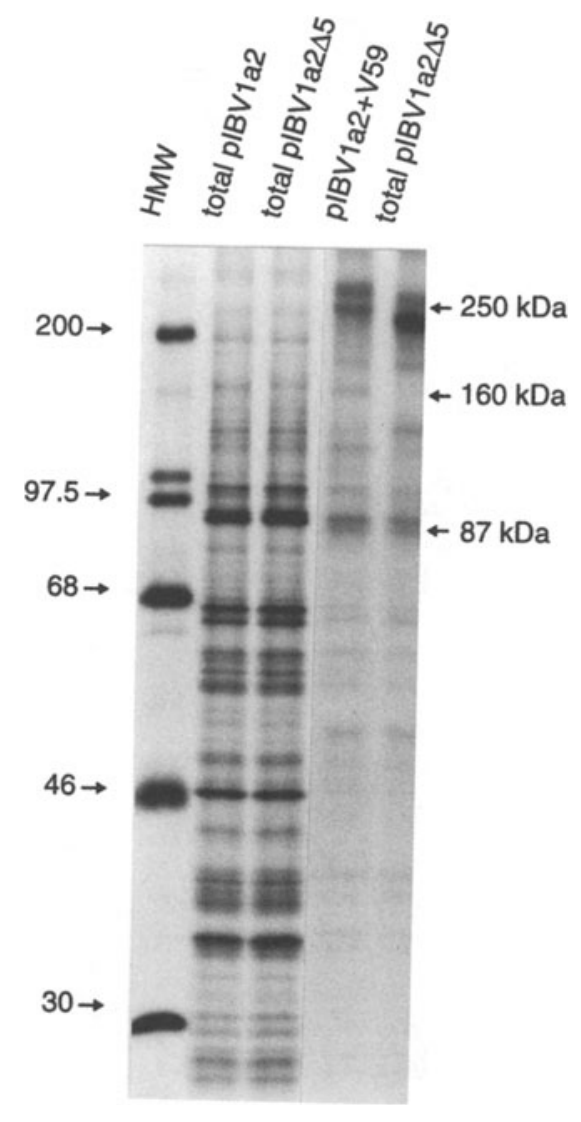


product. We then sought to test if the two predicted residues, Cys ${ }^{1274}$ and His ${ }^{1435}$, is associated with the catalytic activity of the proteinase (Lee et al., 1991). To facilitate this study, plasmid pIBV1a2 $\Delta 4$ (Fig. 1), containing an internal deletion of $0.96 \mathrm{~kb}$ from nucleotide 3047 to 3997, was constructed and used to make the mutant constructs. By monitoring the production of the $87 \mathrm{kDa}$ protein in vivo, we were able to test the effect of the mutation on the proteinase activity. Using pIBV1a $2 \Delta 4$ as the template, the putative nucleophilic residue Cys ${ }^{1274}$ was mutated to a Ser residue (pIBV1a2 $\Delta 4^{\mathrm{C} 1274-\mathrm{S}}$ ) and the predicted His ${ }^{1435}$ residue was mutated to a Lys (pIBVla $2 \Delta 4^{\mathrm{H} 1435-\mathrm{K}}$ ). Fig. 3 shows that expression of pIBV1a2 $\Delta 4$ yielded both the $190 \mathrm{kDa}$ full length polypeptide and the $87 \mathrm{kDa}$ protein. However, expression of pIBV1a2 $\Delta 4^{\mathrm{Cl} 274-\mathrm{S}}$ and pIBV1a2 $\Delta 4^{\mathrm{H} 1435-\mathrm{K}}$ resulted in the detection of only the $190 \mathrm{kDa}$ full-length product by antiserum V59 (Fig. 3). No $87 \mathrm{kDa}$ protein was detected from the two mutant constructs (Fig. 3). This result demonstrated that substitutions of Cys $^{1274}$ with a Ser and $\mathrm{His}^{1435}$ with a Lys disrupt the proteinase activity required for the re-

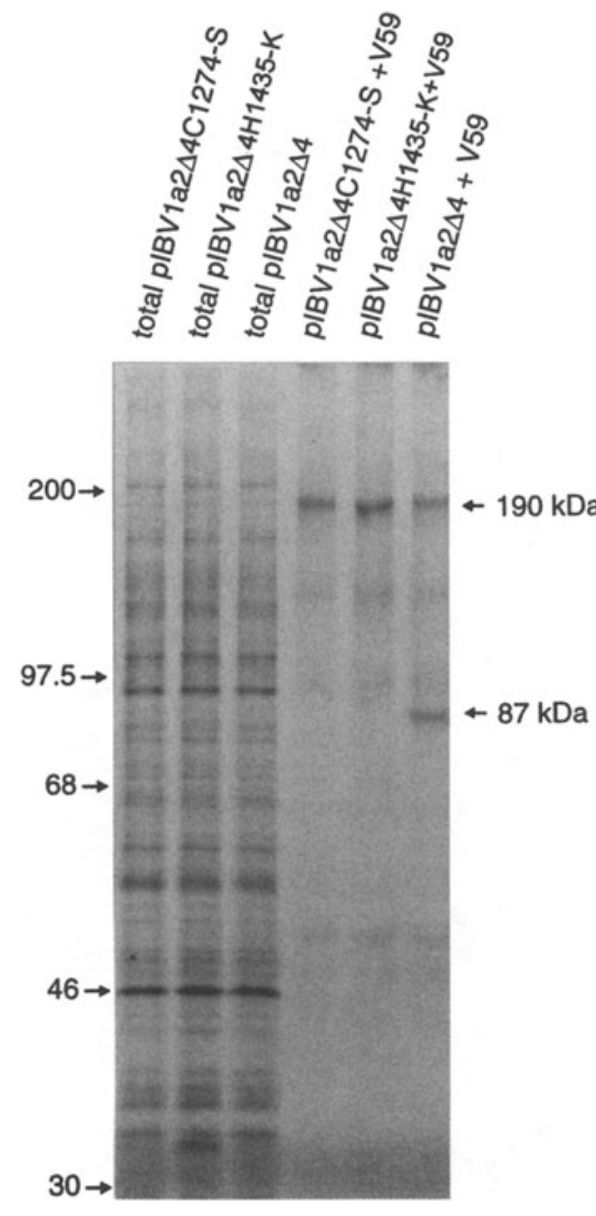

Figure 3. Mutational analysis of the putative catalytic residues Cys ${ }^{1274}$ and His ${ }^{1435}$ of PLPD-1. Plasmids pIBV1a2 $\Delta 4, \quad$ pIBV $1 \mathrm{a} 2 \Delta 4^{\mathrm{C} 1274-\mathrm{S}}$ and pIBV $1 \mathrm{a} 2 \Delta 4^{\mathrm{H} 1435-\mathrm{K}}$ were transiently expressed in Vero cells using the vaccinia recombinant virusT7 expression system. The plasmid transfected cells were labelled with $\left[{ }^{35} \mathrm{~S}\right]$ methionine and the lysates were subjected to immunoprecipitation with V59. The polypeptides were separated on an SDS- $10 \%$ polyacrylamide gel. HMW-high molecular weight markers (numbers indicate kilodaltons). 
lease of the $87 \mathrm{kDa}$ protein. It is therefore suggested that the Cys ${ }^{1274}$ and $\mathrm{His}^{1435}$ may be core residues of the catalytic centre of PLPD-1.

\subsection{Deletion Analysis of the C-Terminal Cleavage Site of the $87 \mathrm{kDa}$ Protein}

The target cleavage site of PLPD-1 was next analysed by deletion analysis. The first deletion mutant, pIBV1a2 $\Delta 8$, was constructed by introducing a UAA termination codon into pIBV1a2 at the nucleotide position 2856. Expression of this plasmid led to the synthesis of a product with an apparent molecular mass of $93 \mathrm{kDa}$, which appeared much larger than the expected size of $87 \mathrm{kDa}$ in SDS-PAGE (Fig. 4a). This implies that the actual cleavage site responsible for releasing the $\mathrm{C}$-terminus of the $87 \mathrm{kDa}$ protein is located at least $250 \mathrm{bp}$ upstream of this position.

To rule out the possibility that the size difference observed was caused by $\mathrm{N}$-terminal cleavage of the pIBV1a2-encoded polyprotein, pIBV1a $2 \Delta 1$ was constructed by deletion of the IBV sequence from nucleotide 362 to 1310. Expression of this construct resulted in the detection of three protein species of $220 \mathrm{kDa}, 160 \mathrm{kDa}$ and $58 \mathrm{kDa}$, representing the full-length and the cleavage products, respectively (Fig. $4 \mathrm{~b}$ ). The migration of the $58 \mathrm{kDa}$ protein in SDS-PAGE suggested that it may be the 5'-truncated version of the $87 \mathrm{kDa}$ protein. Based on pIBV $1 \mathrm{a} 2 \Delta 1$, plasmids pIBV $1 \mathrm{a} 2 \Delta 9$, pIBV1a $2 \Delta 10$, pIBV1a2 $\Delta 11$ and pIBV1a2 $\Delta 12$ (Fig. 1) were then constructed by inserting the UAA stop codon into pIBV1a2 $\Delta 1$ at nucleotide positions $2856,2601,2547$ and 2517 respectively, and were expressed in Vero cells. As shown in Fig. 4b, transfection of pIBV1a $2 \Delta 9$ and pIBV1a $2 \Delta 10$ resulted in the synthesis of the full-length stop products migrating in SDS-PAGE more slowly than the $58 \mathrm{kDa}$ protein. Their approximate sizes were $65 \mathrm{kDa}$ and $61 \mathrm{kDa}$, respectively. Plasmids pIBV1a2 $\Delta 11$ and pIBV1a2 $\Delta 12$ encoded proteins with apparent molecular masses of $58 \mathrm{kDa}$ and $52 \mathrm{kDa}$. The product encoded by pIBVla $2 \Delta 11$ had an electrophoretic mobility in SDS-PAGE almost identical to that of the $58 \mathrm{kDa}$ protein encoded by pIBV1a2 $\Delta 1$ (Fig. 4b). As the UAA termination codon in pIBV1a2 $\Delta 11$ was inserted immediately downstream of the deduced amino acid position 673 , it is likely that the C-terminal cleavage site of the $87 \mathrm{kDa}$ protein is in the vicinity of this residue.

\subsection{Interaction of the $87 \mathrm{kDa}$ Protein with Products Encoded by the Papain-Like Proteinase Domains}

When pIBV1a2 and its derivatives were expressed in vivo, detection of a $160 \mathrm{kDa}$ protein was consistently observed (Fig. 2 and see Liu et al., 1995). It was previously postulated that the $160 \mathrm{kDa}$ protein was an intermediate cleavage product containing the $87 \mathrm{kDa}$ protein, as it could be immunoprecipitated by antiserum V59. However, this possibility was ruled out by the expression of the $\mathrm{N}$ - and $\mathrm{C}$ - terminal deletion constructs pIBV1a2 $\Delta 1$ and pIBV1a2 $\Delta 5$. Expression of pIBV1a2 $\Delta 1$ showed the detection of the $160 \mathrm{kDa}$ protein (Fig. $4 \mathrm{~b}$ ). Expression of pIBV1a2 $\Delta 5$, however, resulted in the synthesis of a $136 \mathrm{kDa}$ product; no $160 \mathrm{kDa}$ protein was detected (see Fig. 2). It is therefore likely that the $160 \mathrm{kDa}$ product was a $\mathrm{C}$-terminal cleavage product released from the $250 \mathrm{kDa}$ polyprotein encoded by pIBV1a2. Detection of the $160 \mathrm{kDa}$ protein by the N-terminal specific antiserum V59 may be due to the interaction of this protein with the $87 \mathrm{kDa}$ protein species.

To investigate this possibility, C-terminal specific antiserum V16 which recognised the IBV sequence between nucleotides 4864 and 5576, was used in immunoprecipitation 


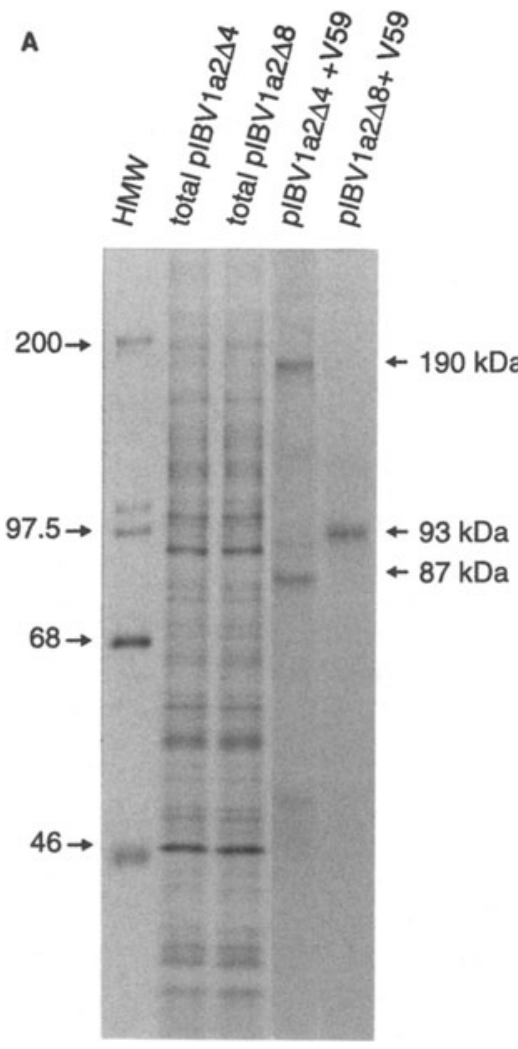

Figure 4. Determination of the $\mathrm{C}$-terminal cleavage site of the $87 \mathrm{kDa}$ protein by deletional studies. A) In vivo expression of pIBV1a $2 \Delta 8$ and pIBV1a2 $\Delta 4$ in Vero cells using the vaccinia recombinant virus-T7 expression system. The cells were labelled with $\left[{ }^{35} \mathrm{~S}\right]$ methionine and the plasmid-transfected cell lysates were subjected to immunoprecipitation with antiserum V59 as indicated above each lane. The proteins synthesised were separated on an SDS- $10 \%$ polyacrylamide gel. HMW-high molecular weight markers (numbers indicate kilodaltons). B) Immunoprecipitation of the proteins encoded by pIBV1a2 $\Delta 1$, pIBV1a2 $\Delta 9$, pIBV1a2 $\Delta 10$, pIBV1a2 $\Delta 11$, and pIBV $1 \mathrm{a} 2 \Delta 12$ were done with V59.

studies. As shown in Figure 5a, immunoprecipitation of pIBV1a2 $\Delta 1$-transfected cell lysate with V16 led to the detection of the $220 \mathrm{kDa}, 160 \mathrm{kDa}$ and $58 \mathrm{kDa}$ protein species. The same three protein species could be immunoprecipitated by V59 (Fig. 5a).

To test the specificities of antisera V59 and V16, plasmids pIBV1a2 $\Delta 8$ (covers the IBV sequences between nucleotides 362 and 2856) and pIBV2P (contains the IBV sequences between nucleotides 3832 and 5757) was expressed in vitro. As can be seen from Figure $5 \mathrm{~b}, \mathrm{pIBV} 1 \mathrm{a} 2 \Delta 8$ encoded a polypeptide with an apparent molecular weight of 93 $\mathrm{kDa}$. This protein could only be immunoprecipitated by antiserum V59 (Fig 5b). Similarly, pIBV2P encoded a $74 \mathrm{kDa}$ protein, which could only be recognised by antiserum V16 (Fig. 5b).

\section{DISCUSSION}

A number of positive-stranded RNA viruses employs proteolytic processing as one of the strategies for their gene expression. Post-translational processing of the $741 \mathrm{kDa}$ polyprotein encoded by the $27.6 \mathrm{~kb}$ genome-length mRNA 1 of the Coronavirus IBV into 


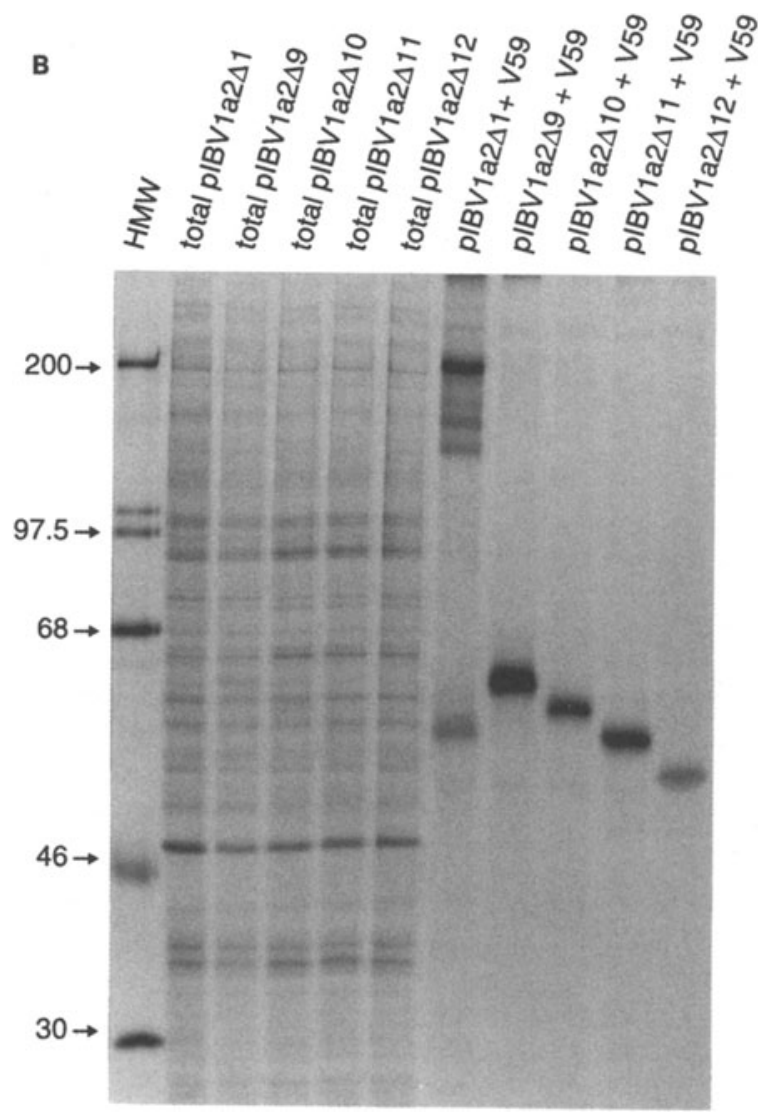

Figure 4B.

smaller functional proteins is probably mediated by both viral and cellular proteinases. One of such cleavage product is an $87 \mathrm{kDa}$ protein identified previously from IBV-infected Vero cells by using a region-specific antiserum V59 (Liu et al., 1995). Data obtained from expression of IBV sequence up to nucleotide 5753 have shown that inclusion of the predicted sequence encoding the two putative overlapping papain-like proteinase domains was required for releasing the $87 \mathrm{kDa}$ protein from the full length precursor. Through the expression of a construct lacking the second of the overlapping papain-like proteinase domains, we demonstrated that this domain was not essential for the in vivo processing of the 1a polyprotein to the $87 \mathrm{kDa}$ product. Therefore, only PLPD-1 encoded between nucleotides 4242 and 5031 was required for the cis -cleavage of the $87 \mathrm{kDa}$ protein from the 1a polyprotein precursor. Site-directed mutagenesis studies confirmed the previously predicted Cys ${ }^{1274}$ and His ${ }^{1435}$ residues as members of the catalytic centre of PLPD-1. The lack of proteolytic activity when either of the catalytic residues was substi- 


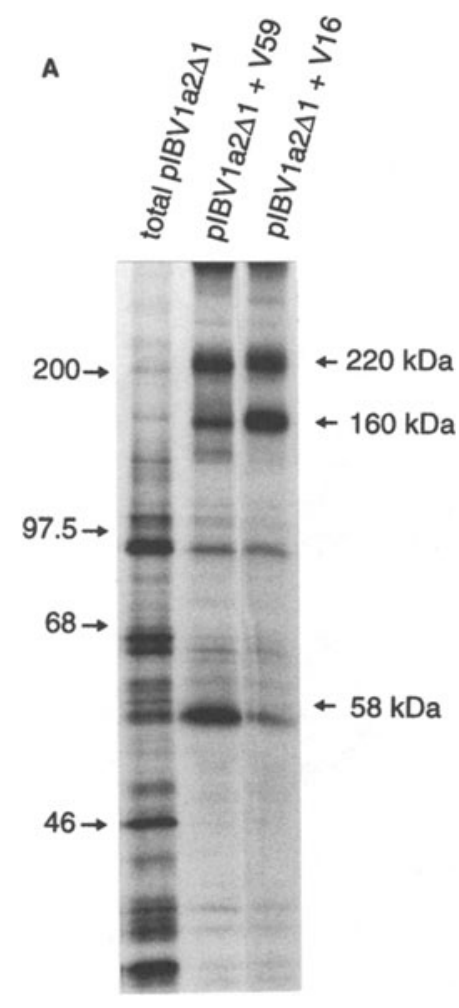

Figure 5. A) Analysis of polypeptides synthesised from in vivo expression of pIBV $1 \mathrm{a} 2 \Delta 1$. The cells were labelled with $\left[{ }^{35} \mathrm{~S}\right]$ methionine and the lysate was subjected to immunoprecipitation with antisera V59 and V16 as indicated above each lane. Electrophoresis of the polypeptides were performed on an SDS-10\% polyacrylamide gel. HMW-high molecular weight markers (numbers indicate kilodaltons). B) Immunoprecipitation of in vitro translation products of RNAs cotranscribed from $\mathrm{pIBV} 1 \mathrm{a} 2 \Delta 8$ and pIBV2P in reticulocyte lysate using the transcription-coupled-translation system (Promega). Approximately $200 \mu \mathrm{g} / \mathrm{ml}$ of plasmid DNA was added to reticulocyte lysate and the proteins were labelled with $\left[{ }^{35} \mathrm{~S}\right]$ methionine. The polypeptides were separated on an SDS- $10 \%$ PAGE either directly or after immunoprecipitation with antisera V59 and V16 and detected by autoradiography. Numbers indicate molecular mass in kilodaltons.

tuted even though the second proteinase domain was still intact further reinforces the conclusion that only the first PLPD is required for the processing to the $87 \mathrm{kDa}$ protein.

Unlike MHV, processing of the ORF1a polyprotein into smaller products by the PLPDs was not observed during the in vitro translation of constructs containing this region of ORF1a (Liu et al., 1995). Microsequencing of the C-terminal cleavage site of the 87 $\mathrm{kDa}$ protein could not be performed as insufficient templates could be generated by using the in vivo expression system. In order to define the C-terminal boundary of the $87 \mathrm{kDa}$ protein, we constructed and expressed a series of deletion constructs by introducing a UAA triplet at different positions of the IBV sequences. By comparing the migration of the termination products in SDS-PAGE with the cleavage product, this approach allows us to define $\mathrm{Gly}^{673}$ - $\mathrm{Gly}^{674}$ dipeptide as a potential cleavage site for releasing the C-terminus of the $87 \mathrm{kDa}$ protein. Mutagenesis studies are underway to confirm this speculation.

We have previously reported that the $160 \mathrm{kDa}$ species is probably the intermediate cleavage product covering the $87 \mathrm{kDa}$ protein, as it could also be immunoprecipitated by region-specific antiserum V59. By construction and expression of $\mathrm{N}$ - and $\mathrm{C}$-terminal deletion constructs, we showed that the $160 \mathrm{kDa}$ protein species was actually not encoded from the 5'-end of ORF $1 \mathrm{a}$, as the full-length $160 \mathrm{kDa}$ protein was observed even when the 5 '-end of ORF1a up to nucleotide 1311 was deleted. Therefore, the $160 \mathrm{kDa}$ protein species probably represents the $\mathrm{C}$-terminal cleavage product resulted from cleavage of the 


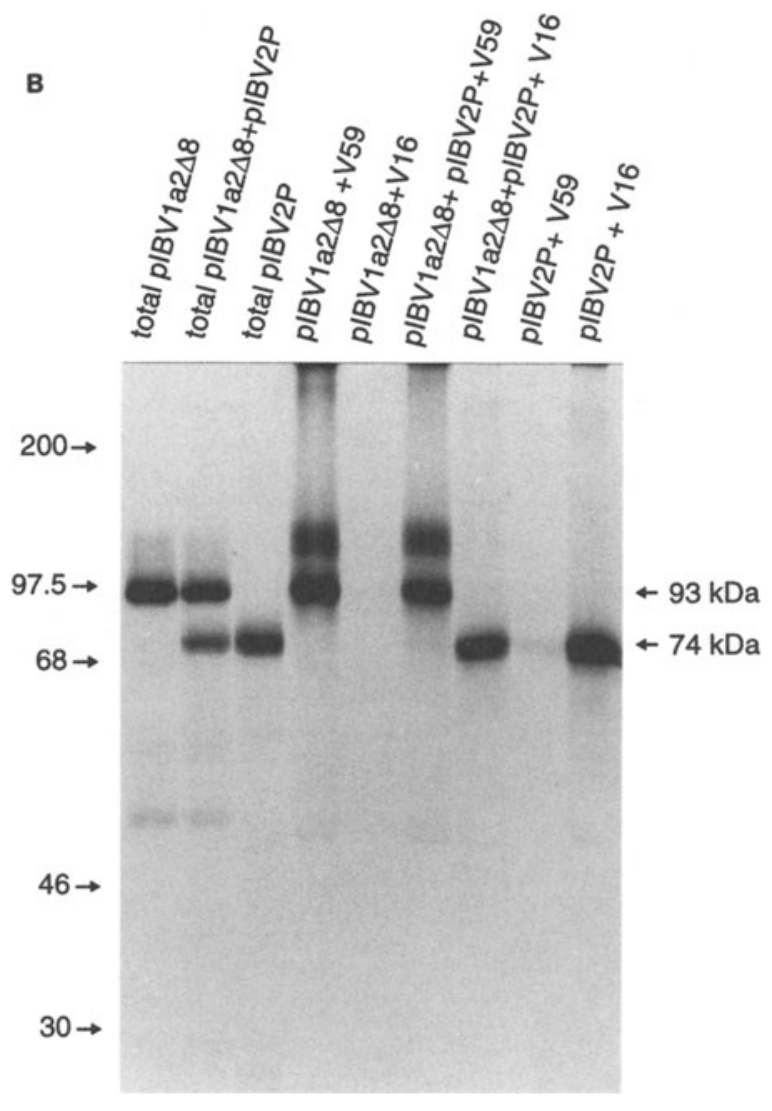

Figure 5B.

$250 \mathrm{kDa}$ polypeptide encoded by pIBV1a2, at the $\mathrm{Gly}^{673}-\mathrm{Gly}^{674}$ dipeptide bond. This result meanwhile rules out the possibility that a second PLPD cleavage site may be located in this portion of the la polyprotein.

Evidence presented confirmed that the detection of the $160 \mathrm{kDa}$ protein by $\mathrm{N}$-terminal specific antiserum is due to the interaction of the $160 \mathrm{kDa}$ protein with the $87 \mathrm{kDa}$ protein. We are currently uncertain if this interaction is essential for the functions of the products encoded by this region of the viral RNA.

\section{REFERENCES}

Boursnell, M. E. G., T. D. K. Brown, I. J. Foulds, P. F. Green, F. M. Tomley and M. M. Binns., 1987, Completion of the sequence of the genome of the coronavirus avian infectious bronchitis virus, J. Gen. Virol. 68:57-77.

Fuerst, T. R., E. G. Niles, F. W. Studier and B. Moss., 1986, Eukaryotic transient-expression system based on recombinant vaccinia virus that synthesises bacteriophage T7 RNA polymerase, Proc. Natl. Acad. Sci. USA 83:8122-8127. 
Gorbalenya, A. E., E. Y. Koonin, A. P. Donchenko and V. M. Blinov., 1989, Coronavirus genome: prediction of putative functional domains in the non-structural polyprotein by comparative amino acid sequence analysis, Nucleic Acids Research 17:4847-4860.

Hughes, S. A., P. J. Bonilla and S. R. Weiss. 1995. Identification of the murine coronavirus p28 cleavage site, $J$. Virol. 69:809-813.

Laemmli, U. K, 1970, Cleavage of structural proteins during the assembly of the bacteriophage T4. Nature (London) 227:680-685.

Lee, H-J., C-K. Shieh, A. E. Gorbalenya, E. V. Koonin, N. L. Monica, J. Tuler, A. Bagdzhadzhyan and M. M. C. Lai, 1991, The complete sequence (22 kilobases) of murine coronavirus gene 1 encoding the putative proteases and RNA polymerase, Virology 180:567-582.

Liu, D. X., I. Brierley, K. W. Tibbles and T. D. K. Brown., 1994, A 100-kilodalton polypeptide encoded by open reading frame (ORF) lb of the coronavirus IBVis processed by ORF la products, J. Virol. 68:5772-5780.

Liu, D. X., K. W. Tibbles, D. Cavanagh, T. D. K. Brown and I. Brierley., 1995, Identification, expression and processing of an $87 \mathrm{kDa}$ polypeptide encoded by ORFla of the coronavirus infectious bronchitis virus, Virology 208:48-57.

Liu, D. X., H. Y. Xu, T. D. K. Brown., 1997, Proteolytic processing of the coronavirus IBVla polyprotein: identification of a 10-kilodalton polypeptide and determination of its cleavage sites, J. Virol 208:48-57. 